FOUNDATIONS OF COMPUTING AND DECISION SCIENCES

Vol. 42

(2017)

No. 1

DE DE GRUYTER

OPEN

DOI: $10.1515 /$ fcds-2017-0003

ISSN 0867-6356

e-ISSN 2300-3405

\title{
GENERALIZATION OF PAWLAK'S APPROXIMATIONS IN HYPERMODULES BY SET-VALUED HOMOMORPHISMS
}

\author{
Saeed MIRVAKILI *, Seid Mohammad ANVARIYEH ${ }^{\dagger}$, Bijan DAVVAZ $\ddagger$
}

\begin{abstract}
The initiation and majority on rough sets for algebraic hyperstructures such as hypermodules over a hyperring have been concentrated on a congruence relation. The congruence relation, however, seems to restrict the application of the generalized rough set model for algebraic sets. In this paper, in order to solve this problem, we consider the concept of set-valued homomorphism for hypermodules and we give some examples of set-valued homomorphism. In this respect, we show that every homomorphism of the hypermodules is a set-valued homomorphism. The notions of generalized lower and upper approximation operators, constructed by means of a set-valued mapping, which is a generalization of the notion of lower and upper approximations of a hypermodule, are provided. We also propose the notion of generalized lower and upper approximations with respect to a subhypermodule of a hypermodule discuss some significant properties of them.
\end{abstract}

Keywords: approximation space, hypermodule, set-valued homomorphism

\section{Introduction}

The rough set theory, proposed by Pawlak $[26,27]$ as a method for data mining in 1982, has attracted the interest of researchers and practitioners in various field of science and technology. This technique has led to many practical applications in various areas such as, but not limited to, medicine learning, knowledge discovery, economics, finance, engineering and even arts and culture $[18,22,23,27,30,31,32,33,40,41$, 44]. Combined with other complementary concepts such as fuzzy sets, statistics, and logical data analysis, rough sets have been exploited in hybrid approaches to improve

\footnotetext{
*Department of Mathematics, Payame Noor University, Tehran, Iran, saeed_mirvakili@pnu.ac.ir

†Department of Mathematics, Yazd University, Yazd, Iran, anvariyeh@yazd.ac.ir

‡Department of Mathematics, Yazd University, Yazd, Iran, davvaz@yazd.ac.ir
} 
the performance of data analysis tools. Application of intelligent methods in industry become a very challenging issue nowadays and will be of extreme importance in the future. Intelligent methods include, fuzzy sets neural networks genetics algorithms and others techniques known as soft computing. No doubt rough set theory can also contribute essentially to this domain [25].

The basic structure of the rough set theory $[27,29]$ is an approximation space consisting of a universe of discourse and an equivalence relation. It is well known that a partition induces an equivalence relation on a set and vice versa. The properties of rough sets can thus be examined via either partition or equivalence relations. The idea is to approximate a subset of a universal set by a lower approximation and upper approximation in the following manner. A partition of the universe is given. The lower approximation is the union of those members of the partition contained in the given subset and the upper approximation is the union of those members of the partition which have a non-empty intersection with the given subset. The members of the partition or equivalence classes can be formally described by unary set-theoretic operators or by successor functions for upper approximation spaces.

Rough set theory is an important technique to deal with vagueness and granularity in information systems. In rough set theory, relation-based rough sets and covering-based rough sets are two important extensions of the classical rough sets. Covering-based rough sets and formal concept analysis are two complementary tools for data analysis. This study systematically explores their connections in terms of approximation operators, structures and knowledge reduction. Lin [20] proposed a more general framework for the study of approximation operators by using the socalled neighborhood systems from a topological space. In a neighborhood system, each element of a universe is associated with a family of subsets of the universe. This family is called a neighborhood system of the element, and each member in the family is called a neighborhood of the element. Any subset of the universe can be approximated based on neighborhood systems of all elements in the universe, also see $[9,10,33,34]$. In $[4,24,45]$ the concept of a cover of a universe was presented to construct the upper and lower approximations of an arbitrary set. In [4] the authors mainly studied the structure of covers while in [24] the authors examined the relationship between the upper and lower approximation operators and some axioms satisfied by the traditional rough sets.

Some researches studies the algebraic properties of rough sets. Biswas and Nanda [3] applied the notion of rough sets to algebra and introduced the notion of rough subgroups. Kuroki [16] introduced the notion of a rough ideal in a semigroup. Kuroki and Wang [17] gave some properties of the lower and upper approximations with respect to normal subgroups. Mordeson [24] used covers of the universal set to define approximations operators on the power set of given set. Davvaz $[5,6]$ concerned a relationship between rough sets and ring theory and considered a ring as a universal set and introduced the notion of rough ideals and rough subrings with respect to an ideal of a ring [8]. Kazanci and Davvaz [13] introduced the notion of rough prime (primary) ideals and rough fuzzy prime (primary) ideals in a ring and gave some properties of such ideals.

A systemic study of generalized rough sets based on arbitrary binary relations was 
first presented by Yao and Lin [38], who based their study on modal logic, but the binary relations in the theory of Modal logic are restricted to finite universes. Lin gave the axioms for binary relations in [19] by two Axioms; We use the same axioms in Section 4. Note that there are two kinds of mathematical objects, pretopological spaces and binary relations, that behave the same from rough set point of view [21].

A model for generalized rough sets based on a binary relation over two universes, or equivalently a many-valued mapping, were first studied by Wong, Wang and Yao; for more information see the book [39]. Also see [16, 28, 42, 43].

The independence of an axiom set for generalized approximation operators is also an important issue in the axiomatic approach that can be observed in [37].

By considering the notion of $\Gamma$-semihypergroups Anvarieh, Mirvakili and Davvaz introduced a relationship between rough sets and hyperstructures. They defined the notion of rough $\Gamma$-hyper ideals with respect to an $\Gamma$-hyperideal of an $\Gamma$-semihypergroup, which is an extended notion of ideal in semigroup and they gave some properties of the lower and the upper approximations in $\Gamma$-semihypergroups [1].

However, equivalence relations are too restrictive for many applications: for instance, in existing databases, the values of attributes could be either symbolic or real-valued. Rough set theory would have difficulty in handling such valued. To address this issue, several known generalizations of the rough model have been reported in the literature. For instance, the notions of approximations are extended to general binary relations, covering, neighborhood systems, completely distributive lattices, fuzzy lattices, set-valued functions and Boolean algebra [4, 33, 32]. Davvaz introduced the concept of a set-valued homomorphism for groups, which is a generalization of ordinary homomorphism. In $[7,36]$, The concepts of set-valued and strong set-valued homomorphism of a group and of a ring are studied and related properties are investigated. The notions of generalized lower and upper approximation operators, constructed by means of a set-valued mapping, which is a generalization of lower and upper approximations of a ring, are provided. Hooshmandasl et al. [12] introduced some axiomatic systems of lower and upper approximation operators in rough set theory and applied the axiomatic systems of generalized rough sets for definitions of generalized lower and upper approximations with respect to an ideal of a ring.

Rough modules have been investigated by Davvaz and Mahdavipour [11], also see [14]. In [35], the concepts of set-valued homomorphism and strong set-valued homomorphism of a module were introduced. The generalized lower and upper approximation operators, which are generalizations of the lower and upper approximations of a module that are constructed using a set-valued mapping, were presented. The authors proposed generalizing the lower and upper approximations with respect to a submodule of a module.

In applied mathematics we encounter many examples of mathematical objects that can be added to each other and multiplied by scalar numbers. First of all, the real numbers themselves are such objects. Other examples are real-valued functions, the complex numbers, infinite series, vectors in n-dimensional space, and vector-valued functions [11]. So, in this paper we discuss a general mathematical concept, called a hypermodule over a hyperring, which includes all those examples and many others as special cases. One of the primary motivations of this work is to apply the 
axiomatic system for generalized rough sets for defining generalized lower and upper approximations with respect to a subhypermodule of a hypermodule.

This paper is structured as follows. After the introduction, we present some basic definitions and then we study the concepts of set-valued and strong set-valued homomorphisms of a hypermodule as a generalization of a hypergroup (group) and a generalization of a module and investigate some related properties. We also propose the notion of generalized lower and upper approximations with respect to a subhypermodule of a hypermodule and discuss some significant properties of them.

\section{Basic definitions}

In this section, we present the basic definitions of algebraic hyperstructures. Note that in hyperstructures we use hyperoperation. In ordinary algebra the composition of two elements is an element, meanwhile in algebraic hyperstructures the composition of two elements is a set.

Definition 2.1. Let $H$ be a non-empty set and let $\mathcal{P}^{*}(H)$ be the family of all nonempty subsets of $H$. A hyperoperation on $H$ is a map $+: H \times H \longrightarrow \mathcal{P}^{*}(H)$ and the couple $(H,+)$ is called a hypergroupoid. If $A$ and $B$ are non-empty subsets of $H$, then we denote $A+B=\bigcup_{a \in A, b \in B} a+b$.

A hypergroupoid $(H,+)$ is called a semihypergroup if for all $x, y, z$ of $H$, we have $(x+y)+z=x+(y+z)$. A semihypergroup $(H,+)$ is said to be a canonical hypergroup if (1) for every $x, y \in H, x+y=y+x$; (2) there exists $0 \in H$ such that $0+x=x$ for all $x \in H$; (3) for every $x \in H$ there exists a unique element $x^{\prime} \in H$ such that $0 \in x+x^{\prime}$ (we shall write $-x$ for $x^{\prime}$ and we call it the opposite of $x$ ); (4) for every $x, y, z \in H, z \in x+y$ implies $y \in-x+z$ and $x \in z-y$. $[2])$.

Now, we recall the definition of hyperring and hypermodule (for more results, see

Definition 2.2. Let $R$ be a non-empty set and + be a hyperoperation and $\cdot$ be an operation on $R$. $(R,+, \cdot)$ is called a hyperring if $(1)(R,+)$ is a hypergroup, such that $0 \in x+x^{\prime}$; (2) Relating to the multiplication, $(R, \cdot)$ is a semigroup having zero as a bilaterally absorbing element; (3) the multiplication is distributive with respect to the hyperoperation.$+ R$ is a hyperring with identity if there exists $1_{R} \in R$, such that for any $r \in R$, we have $r \in 1_{R} \cdot r$.

Definition 2.3. Let $M$ be a hypergroup. $M$ is said to be a left hypermodule over a hyperring $R$ if there exists

$$
\cdot: R \times M \rightarrow \mathcal{P}^{*}(M) ; \quad(a, m) \mapsto a \cdot m
$$

such that for all $a, b \in R$ and $m_{1}, m_{2}, m \in M$, we have

1) $a \cdot\left(m_{1}+m_{2}\right)=a \cdot m_{1}+a \cdot m_{2}$; 
2) $(a+b) \cdot m=a \cdot m+b \cdot m$;

3) $(a \cdot b) \cdot m=a \cdot(b \cdot m)$.

$M$ is said to be a unitary hypermodule over a hyperring $R$ if for any $m \in M, m \in$ $1_{R} \cdot m$.

Throughout this paper, $M$ is a hypermodule over a hyperring $R$ and ' $R$-hypermodule' (or hypermodule over a hyperring $R$ ) means 'left $R$-hypermodule'.

Let $A$ be a non-empty subset of a left $R$-hypermodule $M$. Then $A$ is called a sub-hypermodule of $M$ if $A$ is itself a hypermodule. A sub-hypermodule $A$ of $M$ is normal in $M$ if $x+A-x \subseteq A$ for all $x \in M$. Let $A$ be a subhypermodule of an $R$-hypermodule $M$. Then the quotient hypergroup $M / A=\{m+A \mid m \in M\}$, with external composition $R \times M / A \rightarrow M / A,(r, m) \rightarrow r m+A$ is an $R$-hypermodule, and it is called the quotient $R$-hypermodule of $M$ by $A$.

Let $A$ and $B$ be two non-empty subsets of $R$ and $S$ be a non-empty subset of $M$. Then, any element of $M$ of the form $r_{1} s_{1}+\ldots+r_{n} s_{n}=\sum_{i=1}^{n} r_{i} s_{i}$ with $r_{1}, \ldots, r_{n} \in I$ and $s_{1}, \ldots, s_{n} \in S$ is a linear combination in $I$, or simply an $I$-linear combination of $S$. We denote the set of all such $I$-linear combinations of $S$ by $I S$.

A non-empty subset $A$ of a hypermodule $M$ is an $R$-subhypermodule (or simply subhypermodule) if $a-b \subseteq A$ and $r a \subseteq A$, for all $a, b \in A$ and $r \in R$.

Let $f$ be a mapping of an $R$-hypermodule $M$ to an $R$-hypermodule $N$ such that $f(x+y)=f(x)+f(y)$ and $f(r x)=r f(x)$, for all $x, y \in M$ and $r \in R$. Then $f$ is called an $R$-hypermodule homomorphism.

\section{Generalized approximation space}

Let $U$ and $W$ be two non-empty universes. A subset $\rho \in \mathcal{P}(U \times W)$ is referred to as a binary relation from $U$ to $W$. The relation $\rho$ is referred to as serial if for all $x \in U$ there exists $y \in W$ such that $(x, y) \in \rho$. If $U=W$, then $\rho$ is referred to as a binary relation on $U . \rho$ is referred to as reflexive if for all $x \in U,(x, x) \in \rho ; \rho$ is referred to as symmetric if for all $x, y \in U,(x, y) \in \rho$ implies $(y, x) \in \rho ; \rho$ is referred to as transitive if for all $x, y, z \in U ;(x, y) \in \rho$ and $(y, z) \in \rho$ imply $(x, z) \in \rho$.

A Pawlak approximation space is an ordered pair $(U, \rho)$, where $U$ is a non-empty finite set of object called the universe and $\rho$ is an equivalence relation on $U$, i.e., $\rho$ is reflexive, symmetric and transitive. For each subset $A$ of $U$, its lower and upper approximations are defined by

$$
\underline{\operatorname{Apr}}_{\rho}(A)=\left\{x \mid[x]_{\rho} \subseteq A\right\} \text { and } \overline{\operatorname{Apr}}_{\rho}(A)=\left\{x \mid[x]_{\rho} \cap A \neq \emptyset\right\},
$$

respectively, where $[x]_{\rho}$ denotes the equivalence class of element $x$ by relation $\rho$. The pair $\operatorname{Apr}_{\rho}(A)=\left(\underline{\operatorname{Apr}}_{\rho}(A), \overline{\operatorname{Apr}}_{\rho}(A)\right)$ is called a rough set of $A$ in $U$.

Let $U$ and $W$ be two non-empty universes. Let $T$ be a set-valued mapping given by $T: U \rightarrow \mathcal{P}(W)$. Then, the triple $(U, W, T)$ is referred to as a generalized approximation space. Any set-valued function from $U$ to $\mathcal{P}(W)$ defines a binary relation from 
$U$ to $W$ by setting $\rho_{T}=\{(x, y) \mid y \in T(x)\}$. Obviously, if $\rho$ is an arbitrary relation from $U$ to $W$, then it can be defined as a set-valued mapping $T_{\rho}: U \rightarrow \mathcal{P}(W)$ by $T_{\rho}(x)=\{y \in W \mid(x, y) \in \rho\}$, where $x \in U$. For any set $A \subseteq W$, a pair of lower and upper approximations, $\underline{T}(A)$ and $\bar{T}(A)$, are defined by

$$
\underline{T}(A)=\{x \in U \mid T(x) \subseteq A\} \quad \text { and } \quad \bar{T}(A)=\{x \in U \mid T(x) \cap A \neq \emptyset\} .
$$

Then pair $(\underline{T}(A), \bar{T}(A))$ is referred to as a generalized rough set, and $\underline{T}$ and $\bar{T}$ are referred to as lower and upper generalized approximation operators, respectively.

From the definitions of lower and upper generalized approximation operators, the following theorem can be easily derived [7, 36, 37].

Theorem 3.1. Let $(U, W, T)$ be a generalized approximation space, its lower and upper generalized approximation operators satisfy the following properties: for all $A, B \in \mathcal{P}(W)$,
(L1) $\underline{T}(A)=\left(\bar{T}\left(A^{c}\right)\right)^{c}$,
(U1) $\bar{T}(A)=\left(\underline{T}\left(A^{c}\right)\right)^{c}$,
(L2) $\underline{T}(W)=U$,
(U2) $\bar{T}(\emptyset)=\emptyset$,
(L3) $\underline{T}(A \cap B)=\underline{T}(A)) \cap \underline{T}(B)$,
(U3) $\bar{T}(A \cup B)=\bar{T}(A) \cup \bar{T}(B)$,
(L4) $A \subseteq B \Rightarrow \underline{T}(A) \subseteq \underline{T}(B)$,
(U4) $A \subseteq B \Rightarrow \bar{T}(A) \subseteq \bar{T}(B)$,
(L5) $\underline{T}(A)) \cup \underline{T}(B) \subseteq \underline{T}(A \cup B)$,
(U4) $\bar{T}(A \cap B) \subseteq \bar{T}(A) \cap \bar{T}(B)$,

where, $A^{c}$ is the complement of $A$. Properties (L1) and (U1) show that $\underline{T}$ and $\bar{T}$ are dual approximation operators.

REMARK 1. Let $(U, W, T)$ be a generalized approximation space, for all $A_{i} \in \mathcal{P}(W)$, where $i \in I$ and $I$ is an index set, we have
(1) $\left.\underline{T}\left(\bigcap_{i \in I} A_{i}\right)=\bigcap_{i \in I} \underline{T}\left(A_{i}\right)\right)$,
(2) $\bar{T}\left(\bigcup_{i \in I} A_{i}\right)=\bigcup_{i \in I} \bar{T}\left(A_{i}\right)$,
(3) $\left.\bigcup_{i \in I} \underline{T}\left(A_{i}\right)\right) \subseteq \underline{T}\left(\bigcup_{i \in I} A_{i}\right)$,
(4) $\bar{T}\left(\bigcap_{i \in I} A_{i}\right) \subseteq \bigcap_{i \in I} \bar{T}\left(A_{i}\right)$

Definition 3.2. Suppose that $\oplus$ is a binary hyperoperation on $W$. Then for any set-valued mappings $T: U \rightarrow \mathcal{P}(W)$ and $S: U \rightarrow \mathcal{P}(W)$, the sum $T \widetilde{\oplus} S$ is defined as set-valued mapping $T \widetilde{\oplus} S(a)=T(a) \oplus S(a)=\{p+q \mid p \in T(a), q \in S(a)\}$ for all $a \in U$.

Definition 3.3. Let $U, W$ and $K$ be non-empty universes. Let $T: U \rightarrow \mathcal{P}(W)$ and $S: W \rightarrow \mathcal{P}(K)$ be two set-valued mappings. Then the set valued mappings $S \widetilde{\circ} T$

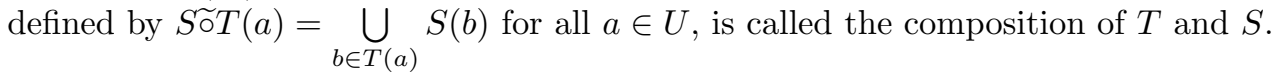




\section{Set-valued homomorphism in hypermodules}

Let $A$ be a subhypermodule of $M$ and $X$ be a non-empty subset of $M$. Then, the sets

$$
\underline{A p r}_{A}(X)=\{a \in M \mid a+A \subseteq X\} \text { and } \overline{A p r}_{A}(X)=\{a \in M \mid a+A \cap X \neq \emptyset\},
$$

where $a+A$ is an element of quotient hypermodule $M / A$, are lower and upper approximations of set $X$ with respect to the subhypermodule $A$. They also gave some properties of the lower and the upper approximations in an $R$-hypermodule.

Rough sets were originally proposed in the presence of an equivalence relation. An equivalence relation is sometimes difficult to be obtained in real-world problems due the vagueness incompleteness of human knowledge. From this point of view, we introduce the concept of set-valued homomorphism of hypermodules and give some examples of set-valued homomorphism. We show that every homomorphism of the hypermodules is a set-valued homomorphism. We also investigate some basic properties of generalized lower and upper approximation operators for hypermodules. First, we give some useful definitions, which will be used in the following sections.

Definition 4.1. Let $M$ and $N$ be two $R$-hypermodules. A mapping $T: M \rightarrow \mathcal{P}(N)$ is called a set-valued homomorphism if and only if

(1) $T(a)+T(b) \subseteq T(a+b)$;

(2) $-T(a) \subseteq T(-a)$;

(3) $r \cdot T(a) \subseteq T(r \cdot a)$;

for all $a, b \in M$ and $r \in R$. A set-valued $T$ is called a strong set-valued homomorphism if we replace inclusion by equality.

By Definition 4.1, it is easy to verify that $T(0)(\neq \emptyset)$ is a subhypermodule of $N$ for every set-valued homomorphism $T$.

ExAmple 1. (1) Let $A$ be a subhypermodule of a hypermodule $M$ and $T: M \rightarrow$ $\mathcal{P}(M)$ be a set-valued function defined by $T(a)=a+A$. Then $T$ is a set-valued homomorphism and $\bar{T}(X)=\overline{A p r_{A}}(X)$ and $\underline{T}(X)=\underline{A p r_{A}}(X)$.

(2) Let $M$ and $N$ be two hypermodules. Then the set-valued mapping $T: M \rightarrow$ $\mathcal{P}(N)$ defined by $T(a)=N$ is a set-valued homomorphism. If $N$ is an unitary $R$-hypermodule, then $T$ is a strong set-valued homomorphism.

(3) Let $M$ and $N$ be two hypermodules. Then the set-valued mapping $T: M \rightarrow$ $\mathcal{P}(N)$ defined by $T(a)=\{0\}$ is a strong set-valued homomorphism.

Proposition 4.2. Let $f: M \rightarrow N$ be an R-hypermodule homomorphism. Then the following hold:

(1) The set-valued mapping $T_{f}: M \rightarrow \mathcal{P}(N)$ defined by $T_{f}(a)=\{f(a)\}$ is a strong set-valued homomorphism such that $\overline{T_{f}}(Y)=f^{-1}(Y)=\underline{T_{f}}(Y)$ for all $Y \in$ $\mathcal{P}(N)$. 
(2) The set-valued mapping ${ }_{f} T: N \rightarrow \mathcal{P}(M)$ defined by ${ }_{f} T(y)=f^{-1}(y)$ is a set valued homomorphism such that for all $X \in \mathcal{P}(M),{ }_{f} T(X)=f(X)$.

(3) If $T: N \rightarrow \mathcal{P}(K)$ is a (strong) set-valued homomorphism, then $T \circ f: M \rightarrow$ $\mathcal{P}(K)$ is a (strong) set-valued homomorphism such that $\overline{T \circ f}(Y)=f^{-1}(\bar{T}(Y))$ and $\underline{T \circ f}(Y)=f^{-1}(\underline{T}(Y))$ for all $Y \in \mathcal{P}(K)$.

(4) If $T: P \rightarrow \mathcal{P}(M)$ is a (strong) set-valued homomorphism, then $F \circ T: P \rightarrow$ $\mathcal{P}(N)$ defined by $F \circ T(a)=F(T(a))$ is a (strong) set-valued homomorphism, where $F: \mathcal{P}(M) \rightarrow \mathcal{P}(N)$ defined by $F(A)=f(A)$ for all $A \in \mathcal{P}(M)$. Additionally, $\overline{F \circ T}(Y)=\bar{T}\left(f^{-1}(Y)\right)$ and $\underline{F \circ T}(Y)=\underline{T}\left(f^{-1}(Y)\right)$ for all $Y \in \mathcal{P}(N)$.

(5) If $A$ is a subhypermodule of $N$, then the set-valued mapping $T_{A}: M \rightarrow \mathcal{P}(N)$ defined by $T_{A}(x)=A+f(x)$ is a set-valued homomorphism.

Proof. The proof is straightforward.

Lemma 4.3. Let $M$ and $N$ be two $R$-hypermodules such that $(M,+)$ be a group. If $T: M \rightarrow \mathcal{P}(N)$ is a set-valued homomorphism and $T(x) \neq \emptyset$, then, $T(x)=T(0)+p$ for every $p \in T(x)$.

Proof. Suppose that $p$ is any element of $T(x)$. If $y \in T(x)$, then $y-p \subseteq T(x)-$ $T(x) \subseteq T(x-x)=T(0)$ and hence $y=y+0=y-p+p \subseteq T(0)+p$. Thus, $T(x) \subseteq T(0)+p$. Conversely, suppose that $z \in T(0)+p$. Then $z-p \subseteq T(0)$ and hence $z=z+0=z-p+p \subseteq T(0)+T(x) \subseteq T(0+x)=T(x)$. This proves that $T(x)=T(0)+p$.

Theorem 4.4. Let $M$ and $N$ be two R-hypermodules such that $(M,+)$ be a group, $0_{R}$. $0_{M}=0_{M}$ and $0_{R} \cdot 0_{N}=0_{N}$. If $T: M \rightarrow \mathcal{P}(N)$ is a strong set-valued homomorphism, then $T(x)=0$ or $T(x)$ is singleton. Specially, if $T: M \rightarrow \mathcal{P}^{*}(N)$, then there exists a hypermodule homomorphism $T: M \rightarrow N$ such that $T=T_{f}$.

Proof. By using the definition of strong set-valued homomorphism, we obtain $T(0)=$ $T(0 \cdot 0)=0 \cdot T(0)=\{0\}$. From Lemma 4.3, we obtain that $T(x)$ has a single element. If $T: M \rightarrow \mathcal{P}(N)$ is a strong set-valued homomorphism, then we define that $f: M \rightarrow N$ by $f(x)=p$, where $T(x)=\{p\}$. Then, it is easily verified that $f$ is a hypermodule homomorphism and $T=T_{f}$.

The proofs of following propositions are similar to the proofs of Propositions 3.6, 3.7 and 3.8 in [35].

Proposition 4.5. Let $M$ and $N$ be two R-hypermodules and $T: M \rightarrow \mathcal{P}(N)$ and $S: M \rightarrow \mathcal{P}(N)$ be two (strong) set-valued homomorphisms. Then $T \tilde{+} S$ is a (strong) set-valued homomorphism.

Proposition 4.6. Let $M, N$ and $K$ be R-hypermodules and $T: M \rightarrow \mathcal{P}(N)$ and $S: N \rightarrow \mathcal{P}(K)$ be two set-valued homomorphisms. Then $S \widetilde{\circ T}$ is a set-valued homomorphism. 
Proposition 4.7. Let $T: M \rightarrow \mathcal{P}(N)$ be a (strong) set-valued homomorphism and $A$ be a subhypermodule of $N$. Define $T / A: M \rightarrow \mathcal{P}(N / A)$ by $T / A(a)=\{x+A \mid x \in$ $T(a)\}$, where $N / A$ is the quotient hypermodule of $N$ by $A$. Then $T / A$ is a (strong) set-valued homomorphism.

ExAmple 2. Let $R$ be a hyperring and $M=R$ and $N=R^{2}$ be two $R$-hypermodules. Let $(a, b) \in R^{2}$ and $T: R \rightarrow \mathcal{P}\left(R^{2}\right)$ be a set-valued function by $T(c)=\{(x, y) \mid c \in$ $a x+b y\}$. Then $T$ is a set-valued homomorphism.

Definition 4.8. [2]. Let $M$ be an $R$-hypermodule and $\theta$ be an equivalence relation on $M$. For every $A, B \subseteq M$ we denote $(A, B) \in \overline{\bar{\theta}}$ if for every $u \in A$ and $v \in B$, we have $(u, v) \in \theta$.

An equivalence relation on $M$ is called a strongly regular relation if for all $a, b \in M$ and $r \in R$, we have the following properties:

(1) $(a, b) \in \theta$ implies $(a+x, b+x) \in \overline{\bar{\theta}}$.

(2) $(a, b) \in \theta$ implies $(r \cdot a, r \cdot b) \in \overline{\bar{\theta}}$.

Example 3. [2]. Let $M$ be an $R$-hypermodule. Let $\theta^{*}$ be the fundamental relation on $M$. Then $\theta^{*}$ is a strongly regular relation on $M$.

Proposition 4.9. Let $\theta$ be a strongly regular relation on a hypermodule $M$. Then $(a, b) \in \theta$ implies $(a+x, b+x) \in \overline{\bar{\theta}}$

(1) $[a]_{\theta}+[b]_{\theta} \subseteq[a+b]_{\theta}$,

(2) $[-a]_{\theta}=-[a]_{\theta}$,

(3) $r \cdot[b]_{\theta} \subseteq[r \cdot b]_{\theta}$

for all $a, b \in M$ and $r \in R$.

Proof. The proof is straightforward.

Corollary 4.10. Let $\theta$ be a strongly regular relation on a hypermodule $M$. Define $T_{\theta}: M \rightarrow \mathcal{P}(M)$ by $T_{\theta}(a)=\{a\}_{\theta}$. Then, $T_{\theta}$ is a set-valued homomorphism.

Proof. The proof follows from Proposition 4.9.

Note that Corollary 4.10 shows that every strongly regular relation may be considerd as a set-valued homomorphism. On the other hand, a strongly regular relation is important in algebraic systems. So, set-valued homomorphism is interesting for pure algebraic systems.

The following examples show that the set-valued homomorphism $T_{\theta}$ in Corollary 4.10 may not be a strong set-valued homomorphism.

EXAMPLE 4 . Let $\mathbb{Z} \times \mathbb{Z}$ be an $\mathbb{Z}$-hypermodule with the scalar hyperoperation $r \cdot(x, y)=$ $\{(0, z) \mid z \in \mathbb{Z}\}$. Consider the relation $\theta$ on $\mathbb{Z} \times \mathbb{Z}$ defined as the follows:

$$
(a, b) \theta(c, d) \Leftrightarrow 4 \mid a-c, b=d .
$$

Then, $\theta$ is a strongly regular relation, but $2 \cdot[(0,0)]_{\theta}=\{(0, z) \mid z \in \mathbb{Z}\}$ and $[2 \cdot(0,0)]_{\theta}=$ $\{(4 k, z) \mid k, z \in \mathbb{Z}\}$. 
Now, we will study some properties of the generalized upper and lower approximation operators by a set-valued homomorphism.

Theorem 4.11. Let $M, N$ be two R-hypermodules and $A$ be a subhypermodule of $N$. Then,

(1) let $T: M \rightarrow \mathcal{P}(N)$ be a set-valued homomorphism. Then $\bar{T}(A)$ is, if it is non-empty, a subhypermodule of $M$,

(2) let $T: M \rightarrow \mathcal{P}(N)$ be a strong set-valued homomorphism. Then $\underline{T}(A)$ is, if it is non-empty, a subhypermodule of $M$.

Proof. (1) Suppose that $x, y \in \bar{T}(A)$ and $r \in R$. Then $T(x) \cap A \neq \emptyset$. Hence, there exist $a \in T(x) \cap A$ and $b \in T(y) \cap A$, which implies that $a-b \subseteq T(x)-T(y) \subseteq$ $T(x-y), a-b \subseteq A$. So, $T(x-y) \cap A \neq \emptyset$. Therefore, $x-y \subseteq \bar{T}(A)$. Also, we have $r \cdot a \subseteq A$ and $r \cdot T(x) \subseteq T(r \cdot x)$. So, $T(r \cdot x) \cap A \neq \emptyset$, which implies that $r \cdot x \subseteq \bar{T}(A)$. Therefore, $\bar{T}(A)$ is a subhypermodule of $M$.

(2) Suppose that $x, y \in \underline{T}(A)$ and $r \in R$. Then, $T(x) \subseteq A$ and $T(y) \subseteq A$. Since $A$ is a subhypermodule of $N$ and $T$ is a strong set-valued homomorphism, we have

$$
T(x-y)=T(x)-T(y) \subseteq A-A \subseteq A \text { and } T(r \cdot x)=r \cdot T(x) \subseteq r \cdot A \subseteq A .
$$

Hence, $x-y, r \cdot x \subseteq \underline{T}(A)$. Therefore, $\underline{T}(A)$ is a subhypermodule of $M$.

Proposition 4.12. Let $M, N$ be two R-hypermodules and $T: M \rightarrow \mathcal{P}(N)$ be a set-valued homomorphism. If $A$ and $B$ are two non-empty subset of $N$ and $I$ is a non-empty subset of $R$, then

1. $\bar{T}(A)+\bar{T}(B) \subseteq \bar{T}(A+B)$;

2. $r \cdot \bar{T}(A) \subseteq \bar{T}(r \cdot A)$, for any $r \in R$;

3. $I \cdot \bar{T}(A) \subseteq \bar{T}(I \cdot A)$;

4. $-\bar{T}(A)=\bar{T}(-A)$.

Proof. (1) Assume that $x$ is any element of $\bar{T}(A)+\bar{T}(B)$. Then $x \in y+z$ with $y \in \bar{T}(A)$ and $z \in \bar{T}(B)$. So, $T(y) \cap A \neq \emptyset$ and $T(z) \cap B \neq \emptyset$. Then, there exist elements $a, b$ such that $a \in T(y) \cap A$ and $b \in T(z) \cap B$. Therefore, $a+b \subseteq A+B$, $a+b \subseteq T(y+z)$, imply $y+z \subseteq \bar{T}(A+B)$ and so $x \in \bar{T}(A+B)$.

The proofs of (2), (3) and (4) are similar to the proof of (1).

Proposition 4.13. Let $T: M \rightarrow \mathcal{P}(N)$ be a strong set-valued homomorphism. If $A$ and $B$ are two non-empty subset of $N$ and $I$ is a non-empty subset of $R$, then

1. $\underline{T}(A)+\underline{T}(B) \subseteq \underline{T}(A+B) ;$

2. $r \cdot \underline{T}(A) \subseteq \underline{T}(r \cdot A)$, for any $r \in R$; 


\section{3. $I \cdot \underline{T}(A) \subseteq \underline{T}(I \cdot A)$;}

4. $-\underline{T}(A)=\underline{T}(-A)$;

Proof. (1) Assume that $x$ be any any element of $\underline{T}(A)+\underline{T}(B)$. Then $x \in y+z$ with $y \in \underline{T}(A)$ and $z \in \underline{T}(B)$. Then, $T(y) \subseteq A$ and $T(z) \subseteq B$. Hence, $T(y+z)=$ $T(y)+T(z) \subseteq A+B$. So, $y+z \subseteq \underline{T}(A+B)$, Therefore, $x \in \underline{T}(A+B)$.

The proofs of (2), (3) and (4) are similar to the proof of (1).

ExAmple 5 . Let $M=\mathbb{Z}$ be a $\mathbb{Z}$-module and $N=\mathbb{Z} \times \mathbb{Z} \times \mathbb{Z}$ be a $\mathbb{Z}$-hypermodule with the scalar hyperoperation $r \cdot(x, y, z)=\{(0, r y, b) \mid b \in \mathbb{Z}\}$. Consider the strong set-valued homomorphism $T: M \rightarrow \mathcal{P}(N)$ defined as $T(a)=\{(0, a, z) \mid z \in \mathbb{Z}\}$. Let $A=\{(0,0, z),(1, z, u) \mid z, u \in \mathbb{Z}\}$ and $B=\{(0,0, z),(-1, z, u) \mid z, u \in \mathbb{Z}\}$. Then, we have $\bar{T}(A)+\bar{T}(B)=\{0\}, \bar{T}(A+B)=\mathbb{Z}, \underline{T}(A)+\underline{T}(B)=\{0\}$ and $\underline{T}(A+B)=\mathbb{Z}$.

Let $(M, N, T)$ be a generalized approximation space. The mapping $T$ is called a lower semiuniform if, for each subhypermodule $A$ in $N$, the set $\underline{T}(A)$ is a subhypermodule of $M$ or empty set. The mapping $T$ is called an upper semiuniform if, for each subhypermodule $A$ in $N$, the set $\bar{T}(A)$ is a subhypermodule of $M$ or empty set. A set-valued mapping $T$ is called a uniform if it is both upper and lower semiuniform.

Corollary 4.14. Every strong set-valued homomorphism is uniform.

Proof. The proof is straightforward by Theorem 4.11 .

ExAmple 6 . Consider $\mathbb{Z}_{4} \times \mathbb{Z}_{4}$ as a $\mathbb{Z}$-hypermodule by defining the scalar hyperoperation $r \cdot(x, y)=\left\{(0, z) \mid z \in \mathbb{Z}_{4}\right\}$ for all $r \in \mathbb{Z}$ and $x, y \in \mathbb{Z}_{4}$. Let $\mathbb{Z}_{4}$ be a $\mathbb{Z}$-module by defining $r \cdot x=0$ for all $r \in \mathbb{Z}$ and $x \in \mathbb{Z}_{4}$. Define the map $T: \mathbb{Z}_{4} \rightarrow \mathcal{P}\left(\mathbb{Z}_{4} \times \mathbb{Z}_{4}\right)$ by $T(0)=T(2)=\{0\} \times \mathbb{Z}_{4} \cup\{2\} \times \mathbb{Z}_{4}$ and $T(1)=T(3)=\{1\} \times \mathbb{Z}_{4} \cup\{3\} \times \mathbb{Z}_{4}$. Then $T$ is uniform, but it is not a strong set-valued homomorphism.

Let $M$ and $N$ be two hypermodules and $A$ be a subset of $N$. Let $T: M \rightarrow \mathcal{P}(N)$ be a set-valued mapping. If $\underline{T}(A)$ and $\bar{T}(A)$ are subhypermodules of $M$, then we call $\underline{T}(A), \bar{T}(A)$ a generalized rough subhypermodule. Note that a generalized rough subhypermodule is also called a generalized rough hypermodule.

The following corollary follows from 4.11.

Corollary 4.15. Let $M$ and $N$ be two hypermodules and $A$ be a subset of $N$.If $T$ : $M \rightarrow \mathcal{P}^{*}(N)$ is a strong set-valued homomorphism, then $(\underline{T}(A), \bar{T}(A))$ is a generalized rough subhypermodule.

\section{Generalized upper and lower homomorphic operator}

Definition 5.1. Let $U$ and $W$ be two universal sets and $\left\{A_{i}\right\}_{i \in I}$ be any collection of subsets of $W$. A mapping $L: \mathcal{P}(W) \rightarrow \mathcal{P}(U)$ satisfying

1. $L(W)=U$, 
2. $L\left(\bigcap_{i \in I} A_{i}\right)=\bigcap_{i \in I} L\left(A_{i}\right)$

is called a lower approximation operator, and a mapping $H: \mathcal{P}(W) \rightarrow \mathcal{P}(U)$ satisfying

1. $H(\emptyset)=\emptyset$,

2. $H\left(\bigcup_{i \in I} A_{i}\right)=\bigcup_{i \in I} H\left(A_{i}\right)$

is called an upper approximation operator.

If $L(A)=\left(H\left(A^{c}\right)\right)^{c}$, or equivalently, $H(A)=\left(L\left(A^{c}\right)\right)^{c}$, for every subset $A$ of $W$, then we say that $H$ and $L$ are dual approximation operators of each other.

ExAmple 7. Let $H: \mathcal{P}(\mathbb{Z}) \rightarrow \mathcal{P}\left(\mathbb{Z}_{m}\right)$ be defined by $H(A)=\left\{r_{m}(x) \mid x \in A\right\}$, where $r_{m}(x)$ is the remainder of $x$ modulo $m$ and $A$ is any subset of $\mathbb{Z}$. Then, $H$ is an upper approximation operator. For example, let $m=7, A_{1}=\{9,8\}$, and $A_{2}=\{8,18\}$. Then, we have $H\left(A_{1}\right)=\{1,2\}, H\left(A_{2}\right)=\{1,4\}$, and $H\left(A_{1} \cup A_{2}\right)=\{1,2,4\}$, i.e., $H\left(A_{1} \cup A_{2}\right)=H\left(A_{1}\right) \cup H\left(A_{2}\right)$.

In the following, the proofs of Theorems 5.2 and 5.4 can be found in Yao's papers (for example, see [39]).

Theorem 5.2. Let $U$ and $W$ be two non-empty universal sets and $L: \mathcal{P}(W) \rightarrow \mathcal{P}(U)$ be a unary operator. Then, there exists a unique set-valued mapping $T: U \rightarrow \mathcal{P}(W)$ such that $L(Y)=\underline{T}(Y)$ for all $Y \subseteq W$ if and only if $L$ is a lower approximation operator.

Corollary 5.3. Let $(U, W, T)$ be a generalized approximation space and the mapping $L: \mathcal{P}(W) \rightarrow \mathcal{P}(U)$ be defined by $L(A)=\underline{T}(A)$, for every $A \subseteq W$. Then, $L$ is a lower approximation operator.

Example 8. Suppose that $L: \mathcal{P}(\{a, b, c\}) \rightarrow \mathcal{P}(\{x, y, z, t\})$ is defined by

$$
\begin{array}{lll}
L(\emptyset)=\emptyset, & L(\{a\})=\emptyset, & L(\{b\})=\{x, z\}, \\
L(\{c\})=\emptyset, & L(\{a, b\})=\{x, z\}, & L(\{a, c\})=\{y\}, \\
L(\{b, c\})=\{x, z\}, & L(\{a, b, c\})=\{x, y, z, t\} . &
\end{array}
$$

We can see that $L$ is a lower approximation operator. By the proof of Theorem 5.2 we can construct the corresponding set-valued mapping $T:\{x, y, z, t\} \rightarrow \mathcal{P}(\{a, b, c\})$ using the formula $T(r)=\left\{s \mid r \notin L\left(s^{c}\right)\right\}$ as follows:

$$
\begin{array}{ll}
T(x)=\{b\}, & T(y)=\{a, c\}, \\
T(z)=\{b\}, & T(t)=\{a, b, c\} .
\end{array}
$$

For the upper approximation, we have the following dual result.

Theorem 5.4. Let $U$ and $W$ be two non-empty universal sets and $H: \mathcal{P}(W) \rightarrow \mathcal{P}(U)$ be a unary operator. Then, there exists a unique set-valued mapping $T: U \rightarrow \mathcal{P}(W)$ such that $H(Y)=\bar{T}(Y)$ for every subset $Y$ of $W$ if and only if $H$ is an upper approximation operator. 
Corollary 5.5. Let $(U, W, T)$ be a generalized approximation space and the mapping $H: \mathcal{P}(W) \rightarrow \mathcal{P}(U)$ be defined by $H(A)=\bar{T}(A)$, for every $A \subseteq W$. Then, $H$ is an upper approximation operator.

Theorem 5.6. Let $L: \mathcal{P}(W) \rightarrow \mathcal{P}(U)$ be a lower approximation operator and $H$ be its dual. Then, for every $A, B \in \mathcal{P}(W)$,

$$
\begin{array}{lll}
\left(L^{\prime} 1\right) & L(A \cap B)=L(A) \cap L(B), & \left(H^{\prime} 1\right) H(A \cup B)=H(A) \cup H(A), \\
\left(L^{\prime} 2\right) A \subseteq B \Rightarrow L(A) \subseteq L(B), & \left(H^{\prime} 2\right) A \subseteq B \Rightarrow H(A) \subseteq H(B), \\
\left(L^{\prime} 3\right) L(A) \cup L(B) \subseteq L(A \cup B), & \left(H^{\prime} 3\right) H(A) \cap H(B) \subseteq H(A \cap B) .
\end{array}
$$

Definition 5.7. Let $M$ and $N$ be two $R$-hypermodules and $H: \mathcal{P}(N) \rightarrow \mathcal{P}(M)$ is an upper approximation operator. The operator $H$ is called a generalized upper homomorphic operator, if for all $I \subseteq R$ and $A, B \subseteq N$,

1. $H(A)+H(B) \subseteq H(A+B)$,

2. $I \cdot H(B) \subseteq H(I \cdot B)$,

3. $-H(A)=H(-A)$,

In the similar way we define a generalized lower homomorphic operator.

Theorem 5.8. Let $R$ be a ring, $M$ be an $R$-hypermodule and $N$ be an $R$-module. If $H: \mathcal{P}(N) \rightarrow \mathcal{P}(M)$ is a generalized upper homomorphic operator, then there is a unique set-valued homomorphism $T: M \rightarrow \mathcal{P}(N)$ such that $H(A)=\bar{T}(A)$.

Proof. According to Theorem 5.4, there exists a unique set-valued mapping $T: M \rightarrow$ $\mathcal{P}(N)$ such that $H(A)=\bar{T}(A)$. We prove that $T$ is a set-valued homomorphism.

1. For all $a, b \in M, T(a)+T(b) \subseteq T(a+b)$. Suppose that $x \in T(a)+T(b)$, then there exist $u \in T(a)$ and $v \in T(b)$ such that $x=u+v$. Hence, $a \in \bar{T}(\{u\})$ and $b \in$ $\bar{T}(\{v\})$, which implies that $a+b \in \bar{T}(\{u\})+\bar{T}(\{v\})$. By condition 1 of Definition 5.7, we have $a+b \subseteq \bar{T}(\{u+v\})$. This result implies that $T(a+b) \cap\{u+v\} \neq \emptyset$. So $u+v \in T(a+b)$. Therefore, $x \in T(a+b)$.

2. For all $r \in R$ and $a \in M$, we show that $r \cdot T(a) \subseteq T(r \cdot a)$. Let $x \in r \cdot T(a)$ then there exists $u \in T(a)$ such that $x=r \cdot u$. Hence, $a \in \bar{T}(\{u\})$ and so by condition 2 of Definition 5.7, we have $r \cdot a \subseteq r \cdot \bar{T}(\{u\})=\bar{T}(\{r \cdot u\})$. This implies that $T(r \cdot b) \cap\{r \cdot u\} \neq \emptyset$. Since $r \cdot u$ is singleton therefore $x=r \cdot u \in T(r \cdot a)$.

3. We show that for every $a \in M,-T(a)=T(-a)$.

$$
\begin{aligned}
u \in-T(a) & \Leftrightarrow-u \in T(a) \\
& \Leftrightarrow a \in \bar{T}(\{-u\}) \\
& \Leftrightarrow-a \in-\bar{T}(\{-u\}) \\
& \Leftrightarrow-a \in \bar{T}(-\{-u\})=\bar{T}(\{u\}), \text { by part }(3) \text { of Definition } 5.7, \\
& \Leftrightarrow u \in T(-a) .
\end{aligned}
$$

The above three results prove that $T$ is a set-valued homomorphism. 
Example 9 . If $N$ is an $R$-hypermodule then Theorem 5.8 is not true.

Let $R=(\mathbb{Z},+, \odot)$ by $x \odot y=0$, then $M=R$ is an $R$-module. Set $N=(\mathbb{Z}, \oplus)$ by $x \oplus y=\left\{\begin{array}{ll}\mathbb{Z} & \text { if } x \neq 0 \neq y, \\ x+y & \text { if } x=0 \text { or } y=0,\end{array}\right.$ for every $x, y \in N$. For every $r \in R$ and $a \in N$ we define $r \cdot a=0$, then $N$ is an $R$-hypermodule. Let $H: \mathcal{P}(N) \rightarrow \mathcal{P}(M)$ defined by $H(A)=A$ for every $A \in \mathcal{P}(N)$. Then $H$ is generalized upper homomorphic operator.

By Theorem 5.4, there exists a unique set-valued mapping $T: M \rightarrow \mathcal{P}(N)$ such that $H(A)=\bar{T}(A)$. So $\bar{T}(A)=A$. We prove that $T(a)=\{a\}$ for every $a \in M$. Since $a \in \bar{T}(a)$ hence $T(a) \cap\{a\} \neq \emptyset$. So $a \in T(a)$. If $b \in T(a)$ then $a \in \bar{T}(b)=\{b\}$. Therefore for every $a \in M, T(a)=\{a\}$. But $T$ is not a set-valued homomorphism. In fact, $T(1) \oplus T(1)=1 \oplus 1=\mathbb{Z}$ and $T(1+1)=\{2\}$.

Theorem 5.9. Let $R$ be a ring, $M$ be an $R$-hypermodule, $N$ be an $R$-module and $L$ : $\mathcal{P}(N) \rightarrow \mathcal{P}(M)$ be an lower approximation operator. If $L$ and its dual are generalized lower and upper homomorphic operators, respectively, then there is a unique strong set-valued homomorphism $T: M \rightarrow \mathcal{P}(N)$ such that $L(A)=\underline{T}(A)$ and $H(A)=\bar{T}(A)$.

Proof. According to Theorem 5.2, there is a unique set-valued mapping $T: M \rightarrow$ $\mathcal{P}(N)$ such that $L(A)=\underline{T}(A)$. We must still prove that $T$ is a strong set-valued homomorphism. By Theorem 5.8, we have $H=\bar{T}$ and $T$ is a set-valued homomorphism. Now,

1. Let $a, b \in M$. Since $T$ is a set-valued homomorphism so $T(a)+T(b) \subseteq T(a+b)$. It is clear that $a \in \underline{T}(T(a))$ and $b \in \underline{T}(T(b))$. Therefore, $a+b \subseteq \underline{T}(T(a))+\underline{T}(T(b))$. By condition 1, we have $a+b \subseteq \underline{T}(T(a)+T(b))$, which implies that $T(a+b) \subseteq$ $T(a)+T(b)$. Therefore, we have $T(a)+T(b)=T(a+b)$.

2. For every $r \in R$ and $a \in M$, we have $r \cdot T(a) \subseteq T(r \cdot a)$. We prove that $T(r \cdot a) \subseteq r \cdot T(a) . a \in \underline{T}(T(a))$ implies $r \cdot a \subseteq r \cdot \underline{T}(T(a))=\underline{T}(r \cdot T(a))$, so $T(r \cdot a) \subseteq T(r \cdot a)$.

3. By the third part of the proof of Theorem 5.8, we have $T(-a)=-T(a)$ for all $a \in M$.

Proposition 5.10. Let $M$ and $N$ be two R-hypermodules. For every subhypermodule $A$ of $N$, the following statements hold:

1. If $H: \mathcal{P}(N) \rightarrow \mathcal{P}(M)$ is a generalized upper homomorphic operator, then $H(A)(\neq \emptyset)$ is a subhypermodule of $M$.

2. If $L: \mathcal{P}(N) \rightarrow \mathcal{P}(M)$ is a generalized lower homomorphic operator, then $L(A)(\neq$ $\emptyset)$ is a subhypermodule of $R$.

Proof. $\quad$ 1. Let $H(A) \neq \emptyset$. Since $A$ is a subhypermodule, then for every $a, b \in H(A)$ and $r \in R$ we have

(i) $a+b \subseteq H(A)+H(A) \subseteq H(A+A) \subseteq H(A)$, 
(ii) $r \cdot a \subseteq r \cdot H(A) \subseteq H(r \cdot A) \subseteq H(A)$,

(iii) $-a \in-H(A) \subseteq H(-A) \subseteq H(A)$.

2. The proof is similar to the proof of (1).

Theorem 5.11. Let $T: M \rightarrow \mathcal{P}(N)$ be a set-valued homomorphism and the mapping $H: \mathcal{P}(N) \rightarrow \mathcal{P}(M)$ be defined by $H(A)=\bar{T}(A)$ for every $A \subseteq N$. Then, $H$ is a generalized upper homomorphic operator.

Proof. We have

1. $H(\emptyset)=\bar{T}(\emptyset)=\emptyset$.

2. $H\left(\bigcup_{i \in I} A_{i}\right)=\bar{T}\left(\bigcup_{i \in I} A_{i}\right)=\bigcup_{i \in I} \bar{T}\left(A_{i}\right)=\bigcup_{i \in I} H\left(A_{i}\right)$, where $\left\{A_{i}\right\}_{i \in I}$ is any collection of subsets of $N$. Thus, $H$ is a generalized upper homomorphic operator.

By Proposition 4.12, for every $A, B \subseteq N$ and $I \subseteq R$, we have

3. $H(A)+H(B)=\bar{T}(A)+\bar{T}(B) \subseteq \bar{T}(A+B)=H(A+B)$,

4. $I \cdot H(A)=I \cdot \bar{T}(A) \subseteq \bar{T}(I \cdot A)=H(I \cdot A)$.

5. $-H(A)=-\bar{T}(A)=\bar{T}(-A)=H(-A)$.

Theorem 5.12. Let $T: M \rightarrow \mathcal{P}(N)$ be a strong set-valued homomorphism and the mapping $L: \mathcal{P}(N) \rightarrow \mathcal{P}(M)$ be defined by $L(A)=\underline{T}(A)$ for every $A \subseteq N$. Then, $L$ is a generalized lower homomorphic operator.

Proof. We have

1. $L(N)=\underline{T}(N)=M$.

2. $L\left(\bigcap_{i \in I} A_{i}\right)=\underline{T}\left(\bigcap_{i \in I} A_{i}\right)=\bigcap_{i \in I} \underline{T}\left(A_{i}\right)=\bigcap_{i \in I} L\left(A_{i}\right)$, where $\left\{A_{i}\right\}_{i \in I}$ is any collection of subsets of $N$. Thus, $H$ is a generalized upper homomorphic operator.

By Proposition 4.13, for every $A, B \subseteq N$ and $I \subseteq R$ we have

3. $L(A)+L(B)=\underline{T}(A)+\underline{T}(B) \subseteq \underline{T}(A+B)=L(A+B)$,

4. $I \cdot L(A)=I \cdot \underline{T}(A) \subseteq \underline{T}(I \cdot A)=L(I \cdot A)$.

5. $-L(A)=-\underline{T}(A)=\underline{T}(-A)=L(-A)$.

Theorem 5.13. Let $T: M \rightarrow \mathcal{P}(N)$ be a set-valued homomorphism and $f: M^{\prime} \rightarrow$ $M$ be an R-hypermodule homomorphism. Define mappings $H, L: \mathcal{P}(N) \rightarrow \mathcal{P}\left(M^{\prime}\right)$ by $H(A)=f^{-1}(\bar{T}(A))$, and $L(A)=f^{-1}(\underline{T}(A))$, for every $A \subseteq N$. Then, $H$ is a generalized upper homomorphic operator, such that $H(A)=\overline{T \circ f}(A)$. If $T$ is a strong set-valued homomorphism then $L$ is a generalized lower homomorphic operator, such that $L(A)=\underline{T \circ f}(A)$. 
Proof. It obtains from Theorems 4.2, 5.8 and 5.9.

Theorem 5.14. Let $H, L: \mathcal{P}(N) \rightarrow \mathcal{P}(M)$ be generalized upper homomorphic and lower homomorphic operators, respectively, and $f: N^{\prime} \rightarrow N$ be a module homomorphism. Then, $H_{f}$ and $L_{f}$ are generalized upper homomorphic and lower strong homomorphic operators defined by $H_{f}(A)=H\left(f^{-1}(A)\right)$ and $L_{f}(A)=L\left(f^{-1}(A)\right)$, respectively.

Proof. It may be obtained from Theorems 4.2, 5.8 and 5.9.

\section{Generalized lower and upper approximation operators with respect to subhypermodule}

In this section, will introduce new generalized lower and upper approximation operators, constructed by means of a set-valued homomorphism, with respect to subhypermodule of a hypermodule which is an extended notation of rough submodule introduced lately by Davvaz and Mahdavipour [11], and we give some basic properties of this new algebraic hyperstructures.

Let $M, N$ be two hypermodules, $A$ be a subhypermodule of $N$ and and $T: M \rightarrow$ $\mathcal{P}(N)$ be a set-valued mapping. Then we define $T_{A}: M \rightarrow \mathcal{P}(N)$ as $T_{A}(x)=T(x)+A$ for all $x \in M . T_{A}$ is called the set-valued mapping with respect to subhypermodule $I$. If $X$ is a non-empty subset of $N$. Then, the sets

$$
\underline{T_{A}}(X)=\{a \in M \mid T(a)+A \subseteq X\} \quad \text { and } \quad \overline{T_{A}}(X)=\{a \in R \mid T(a)+A \cap X \neq \emptyset\}
$$

are called generalized lower and upper approximations of $X$, respectively, with respect to the subhypermodule $A$.

It is easy to see that

1. $\overline{T_{A}}(X)=\bar{T}(X+A)=\overline{T_{A}}(X+A)$,

2. $\underline{T_{A}}(X)=\underline{T}(X+A)=\underline{T_{A}}(X+A)$.

Now, we consider an example of the generalized lower and upper approximations regarding to hypermodules.

EXAMPLE 10. Let $\mathbb{Z} \times \mathbb{Z} \times \mathbb{Z}$ be a $\mathbb{Z}$-hypermodule by the scalar hyperoperation $r$. $(x, y, z)=(r x, r y, \mathbb{Z})$. Set $A=\{(a, a, z) \mid a, z \in \mathbb{Z}\}$, then $A$ is a subhypermodule of $M$. Now, we consider the set-valued homomorphism defined by

$$
T: \mathbb{Z} \rightarrow \mathcal{P}(\mathbb{Z} \times \mathbb{Z} \times \mathbb{Z}), \text { by } T(a)=\{(p, a-p, z) \mid p, z \in \mathbb{Z}\}, \text { for all } a \in \mathbb{Z} .
$$

Assume that $X=\{(1,0, z) \mid z \in \mathbb{Z}\}$ and $Y=\{(x, y, z)|2| x+y\}$, Then the generalized lower and upper approximations of the set $X$ and $Y$ calculated as the follows:

$$
\begin{array}{cc}
\underline{T_{A}}(X)=\emptyset, & \overline{T_{A}}(X)=2 \mathbb{Z}+1, \\
\underline{T_{A}}(Y)=2 \mathbb{Z}, & \overline{T_{A}}(Y)=2 \mathbb{Z} .
\end{array}
$$


Lemma 6.1. Let $A$ and $B$ be two subhypermodules of $N$ such that $A \subseteq B$ and $X$ be a subset of $N$. Then,

1. $\underline{T_{B}}(X) \subseteq \underline{T_{A}}(X)$,

2. $\overline{T_{A}}(X) \subseteq \overline{T_{B}}(X)$.

Proof. The proof is straightforward.

The following corollary follows by Lemma 6.1

Corollary 6.2. Let $A$ and $B$ be two subhypermodules of $N$ and $X$ be a subset of $N$. Then,

1. $\underline{T}_{A}(X) \cap \underline{T}_{B}(X) \subseteq \underline{T}_{(A \cap B)}(X)$,

2. $\bar{T}_{(A \cap B)}(X) \subseteq \overline{T_{A}}(X) \cap \overline{T_{B}}(X)$.

The next example shows that in Corollary 6.2, may equality does not happen:

Example 11. Let $X, Y$ and $A$ be sets defined in Example10 and Let $B=N$ and $C=\{(a, 2 a, z) \mid a, z \in \mathbb{Z}\}$. Then $\underline{T_{A}}(Y) \cap \underline{T_{B}}(Y)=2 \mathbb{Z} \cap \emptyset=\emptyset$ and $\underline{T}_{(A \cap B)}(Y)=2 \mathbb{Z}$. Also $\bar{T}_{(A \cap C)}(X)=\bar{T}_{D}(Y)=\overline{\{1\}}$, where $D=\{(0,0, z) \mid a, z \in \mathbb{Z}\}$, and $\overline{T_{A}}(X) \cap$ $\bar{T}_{C}(X)=(2 \mathbb{Z}+1) \cap(3 \mathbb{Z}+1)=6 \mathbb{Z}+1$.

Theorem 6.3. Let $A$ and $B$ be two subhypermodules of $N$ and $X$ be a subset of $N$.

1. Let $T: M \rightarrow \mathcal{P}(N)$ be a set-valued homomorphism. Let $X$ be a subhypermodule of $N$. Then $\overline{T_{A}}(X)$ is, if it is non-empty, a subhypermodule of $M$.

2. Let $T: M \rightarrow \mathcal{P}(N)$ be a strong set-valued homomorphism and $X$ be a subhypermodule of $N$ such that $A \subseteq X$. Then $\underline{T_{A}}(X)$ is, if it is non-empty, a subhypermodule of $M$.

Proof. (1) Let $a, b \in \overline{T_{A}}(X)$ and $r \in R$. Then $(T(a)+A) \cap X \neq \emptyset$ and $(T(b)+A) \cap X \neq$ $\emptyset$. So, there exist $x \in(T(a)+A) \cap X$ and $y \in(T(b)+A) \cap X$. Since $X$ is a subhypermodule of $N$, we have $x-y \subseteq X$ and $x-y \subseteq(T(a)-T(b))+A \subseteq T(a-b)+A$. Hence, $(T(a-b)+A) \cap X \neq \emptyset$. Also, we have $r \cdot x \subseteq X$ and $r \cdot x \subseteq r \cdot T(a)+r \cdot A \subseteq$ $T(r \cdot a)+A$. So, $(T(r \cdot a)+A) \cap X \neq \emptyset$, which implies that $r \cdot a \in \overline{T_{A}}(X)$. Therefore, $\overline{T_{A}}(X)$ is a subhypermodule of $M$.

(2) Let $a, b \in T_{A}(X)$ and $r \in R$. Then $T(a)+A \subseteq X$ and $T(b)+A \subseteq X$. It is easy to see that $\overline{T(a}-b)+A \subseteq X$. Hence, $a-b \subseteq T_{A}(X)$. Also, we have $T(a) \subseteq X$. So, $T(r \cdot a)=r \cdot T(a) \subseteq r \cdot X \subseteq X$. Since $A \subseteq X$, we have $T(r \cdot a)+A \subseteq X$. Thus, $r \cdot a \subseteq \underline{T_{A}}(X)$. Therefore, $\underline{T_{A}}(X)$ is a subhypermodule of $M$.

Proposition 6.4. Let $A$ be a subhypermodules of $N$ and $X, Y$ be non-empty subset of $N$.

1. If $T: M \rightarrow \mathcal{P}(N)$ is a set-valued homomorphism, then $\overline{T_{A}}(X)+\overline{T_{A}}(Y) \subseteq$ $\overline{T_{A}}(X+Y)$. 
2. Let $T: M \rightarrow \mathcal{P}(N)$ be a strong set-valued homomorphism, $\underline{T_{A}}(X)+\underline{T_{A}}(Y) \subseteq$ $\underline{T_{A}}(X+Y)$.

Proof. (1) Let $x$ be any element of $\overline{T_{A}}(X)+\overline{T_{A}}(Y)$. Then $x \in a+b$ for some $a \in \overline{T_{A}}(X)$ and $b \in \overline{T_{A}}(Y)$. Hence, $(T(a)+A) \cap X \neq \emptyset$ and $(T(b)+A) \cap Y \neq \emptyset$. So, there exist $y \in(T(a)+A) \cap X$ and $z \in(T(b)+A) \cap Y$. Using the definition of $T$ and $X+Y$, we have $y+z \subseteq T(a)+T(b)+A+A \subseteq T(a+b)+A$ and $y+z \subseteq X+Y$. Therefore, $y+z \subseteq(T(a+b)+A) \cap(X+Y)$. Thus, $(T(x)+A)) \cap(X+Y) \neq \emptyset$, which implies that $x \in \overline{T_{A}}(X+Y)$. Hence, $\overline{T_{A}}(X)+\overline{T_{A}}(Y) \subseteq \overline{T_{A}}(X+Y)$.

(2) The proof is similar to the proof of $(1)$.

EXAmPle 12. This example shows that in Corollary 6.2, may equality does not happen. In Example5, Let $A=\{(0,0, z) \mid z \in \mathbb{Z}\}, X=\{(0,0, z),(1, z, u) \mid z, u \in \mathbb{Z}\}$ and $Y=\{(0,0, z),(-1, z, u) \mid z, u \in \mathbb{Z}\}$. Then

$$
\begin{array}{ll}
\overline{T_{A}}(X)+\overline{T_{A}}(Y)=\{0\}, & \overline{T_{A}}(X+Y)=\mathbb{Z}, \\
\underline{T_{A}}(X)+\underline{T_{A}}(Y)=\{0\}, & \underline{T_{A}}(X+Y)=\mathbb{Z} .
\end{array}
$$

Proposition 6.5. Let $A$ and $B$ be two subhypermodules of $M$ and $X$ be a subhypermodule of $N$.

1. If $T: M \rightarrow \mathcal{P}(N)$ is a set-valued homomorphism, then $\overline{T_{A}}(X)+\overline{T_{B}}(X) \subseteq$ $\bar{T}_{A+B}(X)$.

2. Let $T: M \rightarrow \mathcal{P}(N)$ be a strong set-valued homomorphism, $\underline{T_{A}}(X)+\underline{T_{B}}(X)=$ $\underline{T}_{A+B}(X)$.

Proof. (1) Since $A \subseteq A+B$ and $B \subseteq A+B$, then by Lemma 6.1 (2), we have $\overline{T_{A}}(X) \subseteq$ $\bar{T}_{A+B}(X)$ and $\overline{T_{B}}(X) \subseteq \bar{T}_{A+B}(X)$. So, we obtain $\overline{T_{A}}(X)+\overline{T_{B}}(X) \subseteq \bar{T}_{A+B}(X)$.

(2) Since $A \subseteq A+B$ and $B \subseteq A+B$, then by Lemma 6.1 (1), we obtain $\underline{T}_{A+B}(X) \subseteq$ $\underline{T}_{A}(X) \underline{T}_{A+B}(X) \subseteq \underline{T_{B}}(X)$ and so $\underline{T}_{A+B}(X) \subseteq \underline{T}_{A}(X)+\underline{T_{B}}(X)$. Now, let $x \in$ $\overline{T_{A}}(X)+T_{B}(X)$. Then, $x \in a+b$ for some $a \in \overline{T_{A}}(X)$ and $b \in T_{B}(X)$. Hence, $\overline{(T}(a)+A) \subseteq X$ and $(T(b)+B) \subseteq X$. So, $T(x)+A+B \subseteq T(a+b)+A+B=$ $T(a)+T(b)+A+B=T(a)+A+T(b)+B \subseteq X+X=X$, which implies that $x \in \underline{T}_{A+B}(X)$. Therefore, we obtain $\underline{T}_{A}(X)+\underline{T}_{B}(X)=\underline{T}_{A+B}(X)$.

The next example shows that in part (1) of Proposition 6.5, may equality does not happen:

ExAmple 13. Let $M=\mathbb{R}, N=\mathbb{R} \times \mathbb{R}$ and $T(a)=\{(a, a) \mid a \in R\}$. Set $A=0 \times \mathbb{R}$, $B=\mathbb{R} \times 0$ and $X=\{(0,0)\}$. Then

$$
\begin{array}{cc}
\overline{T_{A}}(X)=\{0\}, & \overline{T_{B}}(X)=\{0\}, \\
\overline{T_{A}}(X)+\overline{T_{B}}(X)=\{0\}, & \bar{T}_{A+B}(X)=\mathbb{R} .
\end{array}
$$

Theorem 6.6. Let $M, N$ be two hypermodules and $A$ be a subhypermodule of $N$. If $H: \mathcal{P}(N) \rightarrow \mathcal{P}(M)$ is an upper approximation operator such that $H(X)=H(X+A)$ for every subset $X$ of $N$, then there is a set-valued mapping $T: M \rightarrow \mathcal{P}(N)$ such that $H=\overline{T_{A}}$, i.e., $H$ is a generalized upper approximation operator with respect to subhypermodule $A$. 
Proof. By Theorem 5.4, there is a unique set-valued mapping $T: M \rightarrow P(N)$ such that $H=\bar{T}$. Now, we show that $T_{A}(x)=T(x)$, which implies that $H=\overline{T_{A}}$. It is obvious that $T(x) \subseteq T_{A}(x)=T(x)+A$ for any $x \in M$, because we have $0 \in A$. Let $u$ be an arbitrary element of $T_{A}(x)=T(x)+A$. Then, there exist $y \in T(x)$ and $a \in A$ such that $u \in y+a$, and so $y \in u-a$. Thus, we have $x \in \bar{T}(y)$ or equivalently, $x \in \bar{T}(u-a)$. Therefore, we have $x \in \bar{T}(u+A)$. Because $H=\bar{T}$, by the assumption of the theorem, we obtain $x \in \bar{T}(u)$, which implies that $u \in T(x)$. Hence, $T_{A}=T$.

Definition 6.7. The operator $H$ in Theorem 6.6 is called an upper approximation operator with respect to subhypermodule $A$.

Corollary 6.8. Let $M, N$ be two hypermodules, $A$ be a subhypermodule of $N$, and $H: \mathcal{P}(N) \rightarrow \mathcal{P}(M)$ be a generalized upper approximation operator. If $H_{A}: \mathcal{P}(N) \rightarrow$ $\mathcal{P}(M)$ is defined by $H_{A}(X)=H(X+A)$ for all $A \subseteq S$, then $H_{A}$ is an upper approximation operator with respect to the subhypermodule $A$.

Proof. We have

1. $H_{A}(\emptyset)=H(\emptyset+A)=\emptyset$,

2. for any family $\left\{A_{i}\right\}_{i \in I}$ of subsets of $N$ we have

$$
\begin{aligned}
H_{A}\left(\bigcup_{i \in I} A_{i}\right) & =H\left(\bigcup_{i \in I} A_{i}+A\right)=H\left(\bigcup_{i \in I}\left(A_{i}+A\right)\right) \\
& =\bigcup_{i \in I} H\left(A_{i}+A\right)=\bigcup_{i \in I} H_{A}\left(A_{i}\right),
\end{aligned}
$$

3. $H_{A}(X)=H(X+A)=H(X+A+A)=H_{A}(X+A)$.

Theorem 6.9. Let $H: \mathcal{P}(N) \rightarrow \mathcal{P}(M)$ be a generalized upper approximation operator and $A, B$ be two subhypermodules of $N$ such that $A \subseteq B$. Then, for any non-empty subset $X$ of $N$, we have $H_{A}(X) \subseteq H_{B}(X)$.

Proof. By Corollary 6.8, $H_{A}$ and $H_{B}$ are upper approximation operators with respect to subhypermodules $A$ and $B$, respectively. Additionally, by Theorem 6.6 , there is a set-valued mapping $T: M \rightarrow P(S N)$ such that $H_{A}=\overline{T_{A}}$ and $H_{B}=\overline{T_{B}}$. The remainder of the proof follows from Lemma 6.1 .

Corollary 6.10. Let $H: \mathcal{P}(N) \rightarrow \mathcal{P}(M)$ be a generalized upper approximation operator and and $A, B$ be two subhypermodules of $N$. Then, for any non-empty subset $X$ of $N$, we have $H_{(A \cap B)}(X) \subseteq H_{A}(X) \cap H_{B}(X)$.

\section{Conclusion}

In a classical algebraic structure, the composition of two elements is an element, while in an algebraic hyperstructure, the composition of two elements is a set. On the 
other hand, in applied mathematics we encounter many examples of mathematical objects that can be added to each other and multiplied by scalar numbers. So, in this paper, the concept of rough sets for algebraic hyperstructures such as hypermodules over a hyperring have been concentrated on a congruence relation. In this respect, the notions of generalized lower and upper approximation operators, constructed by means of a set-valued mapping, which is a generalization of the notion of lower and upper approximations of a hypermodule, are provided. We also proposed the notion of generalized lower and upper approximations with respect to a subhypermodule of a hypermodule and discuss some significant properties of them.

\section{References}

[1] S. M. Anvariyeh, S. Mirvakili and B. Davvaz, Pawlak's approximations in $\Gamma$ semihypergroups, Computers and Mathematics with Applications 60 (2010) 4553.

[2] S. M. Anvariyeh and B. Davvaz, Strongly transitive geometric spaces associated to hypermodules, Journal of Algebra, 322 (2009) 1340-1359.

[3] R. Biswas and S. Nanda, Rough groups and subgroups, Bull. Polish Acad. Sci. Math. 42 (1994) 251-254.

[4] Z. Bonikowski, E. Bryniarski, U. Wybraniec-SkardowskaExtensions and intentions in the rough set theory, Inform. Sci. 107 (1998) 149-167.

[5] B. Davvaz, Roughness in ring, Inform. Sci. 164 (2004) 147-163.

[6] B. Davvaz, Roughness based on fuzzy ideals, Inform. Sci. 164 (2004) 2417-2437.

[7] B. Davvaz, A short note on algebraic T-rough sets, Inform. Sci. 178 (2008) 32473252 .

[8] B. Davvaz, Approximations in hyperrings, J. Mult.-Valued Logic Soft Comput. 15 (2009) 471-488.

[9] B. Davvaz, A short note on approximations in a ring by using a neighborhood system as a generalization of Pawlak's approximations, Politehn. Univ. Bucharest Sci. Bull. Ser. A Appl. Math. Phys. 76 (4) (2014) 77-84.

[10] B. Davvaz and M. Mahdavipour, Rough approximations in a general approximation space and their fundamental properties, Int. J. General Systems, 37 (3) (2008) 373-386.

[11] B. Davvaz and Mahdavipour, Roughness in modules, Inform. Sci. 176 (2006) 3658-3674.

[12] M. R. Hooshmandasl, A. Karimi, M. Alamdar and B. Davvaz, Axiomatic system for rough set-valued homomorphisms of associative rings, Int. J. Appr. Reasoning 54 (2013) 297-306. 
[13] O. Kaazanci and B. Davvaz, On the structure of rough prime (primary) ideals and rough fuzzy prime (primary) ideals in commutative rings, Inform. Sci. 178 (2008) 1343-1354.

[14] O. Kazanc1, Sultan Yamak and B. Davvaz, The lower and upper approximations in a quotient hypermodule with respect to fuzzy sets, Inform. Sci. 178(10) (2008) 2349-2359.

[15] M. Kondo, On the srtucture of generalized rough sets, Inform. Sci 176 (2006) 589-600.

[16] N. Kuroki, Rough ideals in semigroups, Inform. Sci 100 (1997) 139-163.

[17] N. Kuroki and P. P. Wang, The lower and upper approximations in a fuzzy group, Inform. Sci. 90 (1996) 203-220.

[18] J. Liang, J. Wang and Y. Qian, A new measure of uncertainty based on knowledge granulation for rough sets., Inform. Sci. 179 (2009) 458-470.

[19] T.Y. Lin, Interpreting association rules in granular data model via decision logic, Annual Meeting of the North American Fuzzy Information Processing Society, Banff, Alberta, Canada, (2004) 57-62.

[20] T.Y. Lin, Q. Liu, K.J. Huang and W. Chen, Rough sets, neighborhood systems and approximation, in: Z.W. Ras, M. Zemankova, M.L. Emrichm (Eds.), Methodologies for Intelligent Systems, Knoxville, Tennessee, 25-27 October 1990, North-Holand, New York, 130-141.

[21] T.Y. Lin, G. Liu, M.K. Chakraborty, D. Slezak, From topology to anti-reflexive topology, Fuzzy Systems (FUZZ), 2013 IEEE International Conference.

[22] D. Q. Miao, Y. Zhao, Y. Y. Yao, H.X. Li and F. F. Xu, Relative reducts in and inconsistent decision tables of the Pawlak rough set model, Inform. Sci. 179 (2009) 4140-4150.

[23] Z. Meng and Z. Shi, A fast approach to attribute reduction in incomplete decision systems with tolerance relation based rough sets, Inform. Sci. 179 (2009) 27742793.

[24] J. N. Mordeson, Rough set theory applied to (fuzzy) ideal theory, Fuzzy Sets and Systems 121 (2001) 315-324.

[25] Z. Pawlak, AI and intelligent industrial applications of the rough set perspective, Journal Cybernetics and Systems, 31(3) (2000) 227-252.

[26] Z. Pawlak, Theoretical Aspects of Reasoning About Data, Kluwer Academic Publishers, Boston, 1991.

[27] Z. Pawlak and A. Skowron, Rudiment of rough sets, Inform. Sci. 177 (1) (2007) 3-27. 
[28] Z. Pawlak and A. Skowron, Rough sets: some extensions, Inform. Sci. 177 (1) (2007) 28-40

[29] Z. Pawlak and A. Skowron, Rough sets and boolean reasoning, Inform. Sci. 177 (1) (2007) 41-73

[30] D. Pei, On definable concepts of rough set models, Inform. Sci 177 (2007) 42304239 .

[31] Y. Qian, J. Liang, D. Li, H, Zhang and C. Dang, Measures for evaluating the decision performance of a decisiontable in rogh set theory, Inform. Sci 177 (2008) 181-202.

[32] W. Z. Wu and W. X. Zhang, Constructive and axiomatic approaches of fuzzy approximation operators, Inform. Sci 159 (2004) 233-254.

[33] W. Z. Wu and W. X. Zhang, Neighborhood operator systems and approximations, Inform. Sci 144 (2002) 201-217.

[34] L. Wang, X. Yang, J. Yang, C. Wu, Relationships among generalized rough sets in six coverings and pure reflexive neighborhood system, Inform. Sci. 207 (2012) 66-78.

[35] S. Yamak, O. Kazanci and B. Davvaz, Approximations in a module by using set-valued homomorphisms, Inter. J. Comp. Math., 14 (2011) 2901-2914.

[36] S. Yamak, O. Kazanci and B. Davvaz, Generalized lower and upper approximations in a ring, Inform. Sci 180 (2010) 1759-1768.

[37] X.-P. Yang, T.-J. Li, The minimization of axiom sets characterizing generalized approximation operators, Inform. Sci. 176 (2006) 887-899.

[38] Y. Y. Yao, T. Y. Lin, Generalization of rough sets using model logic, Intelligent Automation and Soft Computing 2 (1996) 103-120.

[39] Y. Y. Yao, S. K. M. Wong, T. Y. Lin, A review of rough set models, in: T. Y. Lin, N. Cercone (Eds.), Rough Sets and Data Mining: Analysis for Imprecise Data, Kluwer Academic Publishers., Boston, 1997, pp. 47-75.

[40] Y. Yao, There-way decisions with probabilistic rough sets, Inform. Sci. 180 (2010) 341-353.

[41] L. A. Zadeh, Toward a generalized theory of uncertainly (GTU)-An outline, Inform. Sci. 172 (2005) 1-40.

[42] P. Zhu, Covering rough sets based on neighborhoods: An approach without using neighborhoods, Int. J. Approx. Reason. 52 (3) (2011) 461-472.

[43] W. Zhu, F. Wang, Axiomatic systems of generalized rough sets, Proc. RSKT2006, LNAI, Vol. 4062, Springer, 2006, 216-221. 
[44] W. Zhu, Generalized rough sets based on relations, Inform. Sci. 177 (22) (2007) 4997-5011.

[45] W. Zhu, F.-Y. Wang, Reduction and axiomization of covering generalized rough sets, Inform. Sci. 152 (2003) 217230.

Received 2.06.2016, Accepted 13.01.2017 